\title{
New Planetary Nebulae towards the Galactic bulge
}

\author{
P. Boumis ${ }^{1}$, S. Akras ${ }^{1}$, P. A. M. van Hoof ${ }^{2}$, G. C. Van de Steene' ${ }^{2}$, \\ J. Papamastorakis ${ }^{3}$ and J. A. López ${ }^{4}$ \\ ${ }^{1}$ Institute of Astronomy \& Astrophysics, National Observatory of Athens, I. Metaxa \& V. \\ Pavlou, GR-152 36 P. Penteli, Athens, Greece \\ ${ }^{2}$ Royal Observatory of Belgium, Ringlaan 3, B-1180 Brussels, Belgium \\ ${ }^{3}$ Department of Physics, University of Crete, GR-710 03 Heraklion, Crete, Greece \\ ${ }^{4}$ Instituto de Astronoma, UNAM, Apdo. Postal 877, Ensenada, BC 22800, Mexico
}

\begin{abstract}
New Planetary Nebulae (PNe) were discovered through an [O III] $5007 \AA$ emission line survey in the Galactic bulge region with $l>0^{\circ}$. We detected 240 objects, including 44 new PNe. Deep $\mathrm{H} \alpha+[\mathrm{N} \mathrm{II}] \mathrm{CCD}$ images as well as low resolution spectra were obtained for the new $\mathrm{PNe}$ in order to study them in detail. Preliminary photo-ionization models of the new $\mathrm{PNe}$ with Cloudy resulted in first estimates of the physical parameters and abundances. They are compared to the abundances of Galactic PNe.
\end{abstract}

Keywords. surveys, ISM: abundances, ISM: planetary nebulae: general.

\section{Introduction}

Galactic Planetary Nebulae (PNe) are of great interest because of their important role in the chemical enrichment history of the interstellar medium as well as in the stellar evolution of our Galaxy (Beaulieu et al. 2000 and references therein). Many surveys have been made in the past in order to discover new PNe (Boumis et al. 2003 and references therein - Paper I, Boumis et al. 2006 - Paper II, Parker et al. 2006 and references therein).

\section{Observations}

The survey was performed during the 2000-2001 observing seasons with the $0.3 \mathrm{~m}$ telescope at Skinakas Observatory in Crete, Greece. Our aim was to discover PNe which are extended or pointlike showing strong [O III] $5007 \AA$ emission with a signal-to-noise ratio greater than 4. The observational details and the detection method are given in Paper I and Boumis \& Papamastorakis (2001), respectively. Follow-up observations (images and spectra) were obtained with the $1.3 \mathrm{~m}$ telescope at the same site during 2001-2003 and complementary spectral observations during 2005-2006. The images were obtained in $\mathrm{H} \alpha+[\mathrm{N} \mathrm{II}]$ in order to study the morphology of the PNe and also measure their angular extent while their low-resolution spectra confirmed their photo-ionized nature. All new PNe can be seen in Papers I and II.

\section{Preliminary Photoionization Results}

A number of different techniques are in use to determine the physical parameters of PNe. In our case, we decided to use the photo-ionization code Cloudy, last described by Ferland et al. (1998), since this code is widely used and has been tested for many different physical conditions. In this work, we present preliminary results for four of our new PNe. 


\begin{tabular}{llllll|l}
\hline Parameters & PTB17 & PTB26 & PTB31 & PTB34 & mean bulge & mean disk $^{g}$ \\
\hline $\log \left(\mathrm{L} / \mathrm{L}_{\odot}\right)$ & 2.80 & 2.78 & 3.82 & 4.12 & & \\
$\mathrm{~T}_{e f f^{b}}$ & 111.4 & 65.0 & 56.9 & 85.9 & & \\
$\mathrm{n}_{e^{d}}$ & 0.17 & 0.06 & 0.18 & 0.59 & & \\
$\mathrm{~T}_{e^{b}}$ & 9.8 & 6.49 & 9.72 & 8.20 & & \\
$\log \left(\mathrm{M} / M_{\odot}\right)$ & 0.19 & 0.46 & -0.16 & -0.61 & & \\
radius in $^{e}$ & 0.20 & 0.28 & 0.10 & 0.05 & & \\
radius out $^{e}$ & 0.40 & 0.72 & 0.29 & 0.21 & & \\
filling factor & 1.00 & 1.00 & 1.00 & 0.58 & & \\
$\log (\mathrm{U})$ & -2.75 & -2.53 & -1.11 & -0.72 & & \\
${\text { dust } / \text { gas }^{a}}_{\text {distance }^{f}}$ & 7.56 & 6.15 & 6.08 & 5.66 & & \\
$\epsilon(\mathrm{He})$ & 11.17 & 7.80 & 7.80 & 7.00 & & \\
$\epsilon(\mathrm{O})$ & 8.47 & $9.16:$ & 8.35 & 9.06 & 8.66 & \\
$\epsilon(\mathrm{N})$ & 8.12 & 8.01 & 8.32 & 8.34 & 8.43 & 8.34 \\
$\epsilon(\mathrm{S})$ & 6.58 & 6.79 & 7.10 & 7.55 & 7.05 & 6.93 \\
$\epsilon(\mathrm{Ne})$ & - & $8.00^{\dagger}$ & $8.00^{\dagger}$ & - & 8.03 & 8.08 \\
$\epsilon(\mathrm{Ar})$ & - & - & - & 6.96 & 6.60 & 6.42 \\
\hline
\end{tabular}

Table 1. The physical parameters of our sample's PNe determined with Cloudy. (a) $10^{-3}$, (b) $\mathrm{kK},(\mathrm{c}) \log (\mathrm{X} / \mathrm{H})+12$, (d) $10^{3} \mathrm{~cm}-3$, (e) pc, (f) kpc, (g) Exter et al. (2004), ( $\dagger$ ) fixed at an assumed value, (:) the value is uncertain.

Full results will be presented in Akras et al. (2006). The model assumptions can be found in van Hoof \& Van de Steene (1999). It should be noted that as nebular distances we used both the fixed bulge distance $(7.8 \mathrm{kpc})$, and distances determined with the method described in Van de Steene \& Zijlstra (1995). All results will be presented analytically by Akras et al. (2006). The observed quantities used to derive the physical parameters of our PNe are (a) the emission line spectrum of each nebula, (b) their angular diameters and (c) the total hydrogen flux. Further modeling will be performed including radio and/or infrared data whenever they are available. The resulting physical parameters for our sample PNe determined with Cloudy are given in Table 1. We also present the mean bulge and disc abundances taken from Exter et al. (2004).

\section{Acknowledgements}

$\mathrm{AS}$ and $\mathrm{PvH}$ acknowledge financial support from the Belgian Science Policy Office project IUAP P5/36. Skinakas Observatory is a collaborative project of the University of Crete, the Foundation for Research and Technology-Hellas and the Max-Planck-Institut fur Extraterrestrische Physik.

\section{References}

Akras, S., Boumis, P., van Hoof P.A.M., et al. 2006, MNRAS in preparation

Beaulieu, S.F., Freeman, K.C., Kalnajs, et al. 2000, AJ, 120, 855

Boumis, P. \& Papamastorakis, J. 2001, in Proc.5th Hellenic A.S.Conf., astro-ph/0204015

Boumis, P., Paleologou, E.V., Mavromatakis, F., et al. 2003, MNRAS, 339, 735 (Paper I)

Boumis, P., Akras, S., Xilouris, E.M., et al. 2006, MNRAS, 367, 1551 (Paper II)

Exter, K.M., Barlow, M.J. \& Walton, N.A. 2004, MNRAS, 349, 1291

Ferland, G.J., Korista, K.T., Verner, et al. 1998, PASP, 110, 761

Parker Q.A., Acker A., Frew D. J., et al. 2006, MNRAS, submitted

Van de Steene G.C. \& Zijlstra A.A. 1995, A\&A, 293, 541

van Hoof P.A.M. \& Van de Steene G.C. 1999, MNRAS, 308, 623 